\title{
Neutron Polarisation Analysis of Polymer:Fullerene Blends for Organic Photovoltaics
}

\author{
Giuseppe Maria Paternò ${ }^{1}$, J. Ross Stewart ${ }^{2}$, Andrew Wildes ${ }^{3}$, Franco Cacialli ${ }^{1}$ and Victoria García Sakai ${ }^{2}$ \\ ${ }^{1}$ UCL Dept. of Physics and Astronomy and London Centre for Nanotechnology, London WC1H 0AH, UK \\ ${ }^{2}$ ISIS Pulsed Neutron and Muon Source, Science and Technology Facilities Council, Rutherford Appleton Laboratory, \\ Harwell Science and Innovation Campus, Didcot OX11 0QX, UK \\ ${ }^{3}$ Institut Laue-Langevin, 71 avenue des Martyrs - CS 20156 - 38042 Grenoble Cedex 9, France
}

\begin{abstract}
The photogeneration process in polymer-fullerene organic solar cells relies strongly on the nanostructure and on the nano/picosecond dynamics occurring in these complex blends. Elastic and inelastic neutron scattering techniques are valuable tools with which to investigate those features in the appropriate time and space domains. In particular, quasi-elastic neutron scattering (QENS) connects useful structural and dynamical information by the measurement of dynamical incoherent (single particle) fluctuations in soft materials as a function of lengthscale. Extraction of these fluctuation rates can, however, be hampered by the presence of coherent contributions, originating from elastic scattering, and/or inelastic scattering modes which overlap in the space/time domain with the incoherent single-particle motions. As we have already seen in a previous study ${ }^{1}$, this happens in poly(3-hexylthiophene) (P3HT) and [6,6]-Phenyl $\mathrm{C}_{61}$ butyric acid methyl ester (PCBM) solid blends, in which the coherent contribution arising from the PCBM crystalline phase seems to affect the interpretation of the polymer dynamics. Here, we utilise neutron polarisation analysis as an effective tool to separate coherent and incoherent contributions and make QENS data analysis of these blends more reliable.
\end{abstract}




\section{Introduction}

Blends of conjugated polymers and fullerene derivatives are the current paradigm for state-of-the-art polymer photovoltaic diodes. These belong to the class of excitonic solar cells, in which the photocurrent originates from the splitting of the photogenerated bound electron-hole pairs, so-called excitons, which for organic low dielectric constant materials $(\varepsilon=2-3)$ exhibit a relatively high binding energy $^{2}$ and a diffusion length confined to a few monomers $(5-10 \mathrm{~nm})^{3}$. The bulk-heterojunction (BHJ) architecture, in which the formation of an interpenetrated network between the electron-donor and acceptor materials upon blending has the potential to partially overcome these limitations $\mathrm{s}^{4,5}$, enables power conversion efficiencies approaching $10 \%{ }^{6}$. The nanoscale structural/dynamical features of these solid blends, such as the crystallisation of the fullerene molecules ${ }^{7-9}$ as well as the reorganisation of the polymer amorphous and crystalline domains ${ }^{10}$, control the optoelectronic response of the active material and, hence, the efficiency of the photovoltaic process. Therefore, in order to boost the performance of this class of devices, it is important to gain deeper insight into the thermodynamic and kinetic phenomena that occur upon blending and post-process treatments.

Neutron scattering has been employed as a sensitive and non-destructive tool to investigate the structure/dynamic/efficiency relationship in polymer/fullerene blends for organic photovoltaics ${ }^{10-}$ ${ }^{12}$. One advantage of using neutron scattering to study these polymer-nanocomposite systems stems from the strong contrast in scattering between polymer chains and fullerene particles, as the neutron scattering length of fullerene derivatives differs considerably from that of highly hydrogenated conjugated polymers. For instance, if we consider the benchmark blend for BHJ organic solar cells, which is based on regioregular poly(3-hexylthiophene) (P3HT) as the electron donor and [6,6]-Phenyl $\mathrm{C}_{61}$ butyric acid methyl ester (PCBM) as the electron acceptor ${ }^{13}$, the scattering length densities (SLD) are $7 \times 10^{-7} \AA^{-2}$ and $3.6 \times 10^{-6} \AA^{-2}$ for P3HT and PCBM, respectively. Therefore, elastic and inelastic neutron scattering techniques are extremely powerful in mapping a variety of phenomena at the nanoscale, such as polymer-fullerene phase separation and mutual diffusion at their interfaces. Quasielastic neutron scattering (QENS), in particular, is well adapted to the measurement of molecular motions in soft matter over a large dynamical range; such as fast vibrations and rotations as well as slower segmental relaxations and diffusion, with the advantage of mapping their nanoscale spatial dependence as a function of the momentum transfer, $Q^{14}$. As QENS is highly sensitive to hydrogen dynamics due to its high incoherent scattering cross section, the fact that P3HT holds thirteen $\mathrm{H}$ atoms on the side chain and only one on the backbone can be used to highlight the dynamical fluctuations of the side-chain in this functional polymer.

In recent years, QENS has started to be employed in the study of side-chain dynamics of pure poly(alkylthiophenes) ${ }^{15,16}$ and in blends with carbon nanotubes ${ }^{17}$ or fullerene derivatives ${ }^{18}$. In this 
context, we have carried out a QENS study of P3HT-PCBM blends ${ }^{1}$ as a function of blending and solvent choice, using the high-resolution backscattering spectrometer IRIS at the ISIS Neutron and Muon Facility - $\mathrm{UK}^{19}$ in the picoseconds time range. We found that the addition of PCBM frustrates polymer motion, possibly related to polymer confinement within PCBM domains. However, it seems that there is some excess elastic signal fraction at $Q$-values that correspond to the characteristic distance of PCBM crystals (discussed further in the Results section). Calculation of the different contributions to the scattering cross-sections for a 1:1 P3HT:PCBM concentration yields incoherent and coherent contributions of ca. $75.5 \%$ and $5.5 \%$ for P3HT respectively, and $14 \%$ and $5 \%$ for PCBM respectively. Thus, three possible scenarios arise: i) the frustration of P3HT polymer dynamics is real (slowing down or increased elastic fraction), and it is due to P3HT-PCBM interaction and/or polymer confinement within PCBM domains; ii) the increase of the elastic fraction is largely due to the increase of coherent signal caused by PCBM addition and crystallisation; iii) a combination of the effects. It is therefore important to assess whether the excess of coherent contribution at this $Q$-range affects data interpretation. To this end, neutron polarisation analysis can be a powerful method to disentangle collective events from single-particle motions in complex systems. Although this technique has been already employed to discriminate between localised and non-localised motions in ionic liquids ${ }^{20}$, it has never been used to investigate blends of semi-crystalline conjugated polymers with fullerene derivatives.

In the work presented here, we use neutron polarisation analysis to separate the coherent/incoherent scattering contributions in pure P3HT and in blends with PCBM (figure 1), by making use of the diffuse scattering spectrometer D7 at ILL ${ }^{21}$. We observe that, although the polymer motion seems to fall outside the instrumental time-window, this technique can be used effectively to discriminate the coherent/incoherent contributions in a multicomponent polymer:fullerene blend and, in general, in those systems in which the coherent contribution can make data analysis/interpretation non-trivial, i.e. in polymer:nanocomposite systems ${ }^{22}$.

(a)

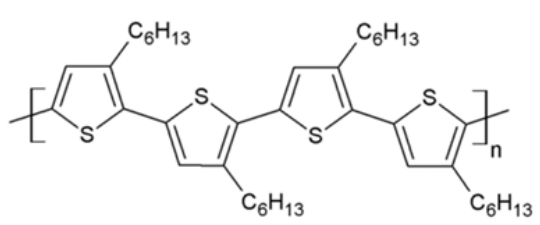

(b)

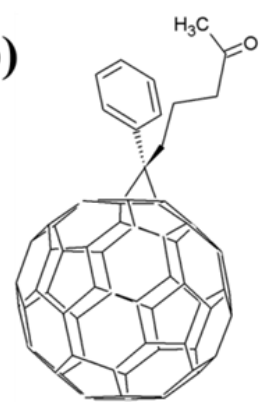

Figure 1: Molecular structure for P3HT (a) and PCBM (b). P3HT and PCBM are the electron-donor and electron-acceptor of choice in bulk heterojunction organic solar cells. 


\section{Materials and Methods}

\subsection{Materials and sample preparation}

Regioregular P3HT $(\mathrm{Mw}=10,000-40,000 \mathrm{~g} / \mathrm{mol})$ and PCBM were purchased from American Dye Source and used without further purification steps. Film of pure P3HT and blends of $50 \mathrm{wt} \%$ P3HT-PCBM were prepared by dissolving the two materials in chloroform separately with a concentration of $20 \mathrm{mg} / \mathrm{mL}$, and mixing them in a 1:1 ratio. The resulting solutions were stirred overnight and drop-cast onto aluminium foil, yielding sample films or around $150 \mu \mathrm{m}$ (as needed for the QENS neutron scattering experiments to achieve transmissions of $\sim 85 \%$ and minimize multiple scattering effects). The samples were vacuum-dried overnight at room temperature (RT $=20-25^{\circ} \mathrm{C}$ ) to remove any residual solvent.

\subsection{D7 experiment}

The diffuse scattering spectrometer D7 measures neutron scattering with polarization analysis and, hence, unambiguously separates collective (nuclear coherent) and single-particle (nuclear incoherent) and magnetic scattering processes by means of $x y z$-polarisation analysis. This is a valid claim in cases where the incoherent scattering is dominantly spin-incoherent (i.e. due to the spin-dependent nuclear scattering length) and not isotope-incoherent (i.e. due to randomly positioned nuclear isotopes). This is a valid assumption for incoherent scattering for most H-containing materials. We used D7 with an incident wavelength of $5.7 \AA$ which covers a similar $Q$-range to that measured on IRIS $\left(0.3-1.8 \AA^{-1}\right)^{1}$. We measured both in diffraction mode, to determine the structure factor of the pure polymer and P3HT:PCBM blends, and in inelastic mode to measure the QENS signal of the incoherent/coherent scattering using the Fermi chopper option. D7 is equipped with a supermirror analyser detector bank with an angular range of $132^{\circ}$. We measured the spin-flip and non-spin-flip scattering with the polarization axis normal to the scattering plane, with the axis preserved by means of a magnetic guide field $(\sim 10 \mathrm{G})$ that extended along the path of incident and scattered beams. The polarization efficiency of the instrument was determined as 0.92 by measuring an amorphous quartz standard that gives only coherent non-spin-flip scattering. The detector efficiency was calibrated using a vanadium standard, and the instrumental energy resolution was estimated from the elastic linewidth of the vanadium (FWHM 0.1 meV). The background contribution was evaluated by measuring the signal of empty cans and scaling it to the sample transmissions. 


\subsection{Neutron polarisation analysis}

The separation of coherent from incoherent scattering using neutron polarization analysis is vital for those systems in which the collective coherent dynamical motions can intrinsically affect the interpretation of the scattering data, i.e., in cases where the coherent scattering cross-sections are not negligible in comparison to the incoherent scattering cross-sections. The key principle of neutron polarisation analysis is that in the case of spin-incoherent scattering the neutron spin is flipped with a 2/3 probability, whereas for coherent (plus isotope-incoherent) scattering no such spin-flip occurs. The scattering intensities for neutron spin-flip ( $\downarrow$ ) and non-spin-flip ( $\uparrow)$ in the limit of zero isotopeincoherent scattering are given by ${ }^{23,24}$ :

$$
I_{\uparrow \uparrow}=I_{c o h}+\frac{1}{3} I_{i n c}
$$

and

$$
I_{\uparrow \downarrow}=\frac{2}{3} I_{i n c}
$$

and therefore the coherent and incoherent intensities can be separated according to:

$$
\begin{gathered}
I_{\text {coh }}=I^{\uparrow \uparrow}-\frac{1}{2} I^{\uparrow \downarrow} \\
I_{\text {inc }}=\frac{3}{2} I^{\uparrow \downarrow}
\end{gathered}
$$

\subsection{Data analysis}

The data were reduced using the LAMP $^{25}$ suite of programs at the ILL and analysed using the DAVE $^{26}$ software package. The quantity that is measured in a QENS experiment is the doubledifferential scattering cross section $\mathrm{d}^{2} \sigma / \mathrm{d} E \mathrm{~d} \Omega$, which determines the probability that a neutron is scattered with an energy change $\mathrm{d} E$ into the solid angle $\mathrm{d} \Omega$. The scattering events can be divided into three main categories: i) when $\mathrm{d} E=0$ or below the energy resolution of the instrument, the scattering event is called elastic; ii) if $\mathrm{d} E \neq 0$ the event is called inelastic; iii) if $\mathrm{d} E$ is close to zero we observe a broadening around the elastic line and the scattering is known as quasi-elastic; such a broadening may be due to a variety of dynamical processes such as zone-centre lattice excitations, vibrations, rotations and translations. The scattering signal in our system is dominated by the incoherent scattering of the hydrogenated side-chains of P3HT plus a small contribution due to PCBM side-group, as a consequence, any motion observed arises mainly from the dynamics of the polymer side chain. 
However, both P3HT and PCBM have measurable coherent contributions that, especially in the $Q$ range around the Bragg peaks, can make structural/dynamical data difficult to interpret. For the evaluation of the QENS signal, the instrumental resolution was measured at $2 \mathrm{~K}$ for each sample. The best fit to the measured dynamical structure functions $S(Q, \omega)$ was obtained with a delta function that accounts for elastic events, and two Lorentzian functions to describe the quasi-elastic broadening. One Lorentzian can be related to fast-motions $(\sim 1 \mathrm{meV})$ and the other one to slower motions $(\sim 0.1 \mathrm{meV})$. Fitting with a delta plus one Lorentzian did not provide a satisfactory fit. The elastic fraction was calculated from the areas of delta and Lorentzian function and plotted as a function of momentum transfer vector $Q$.

\section{Results and discussion}

\subsection{Diffraction with polarisation analysis}

We used D7 in diffraction mode to determine the structure factor of pure P3HT and P3HT-PCBM blends. Figure 2 shows the coherent and incoherent signal contribution to the total diffraction pattern for pure P3HT (a) and in a blend with PCBM (b) separated using neutron polarisation analysis. We can observe that the diffraction pattern is clearly modulated by the coherent contribution that originates mainly from the polymer and PCBM crystalline domains. Regioregular P3HT (figure 2a) crystallises into lamellar structures, which can adopt two kinds of orientation with respect to the substrate plane: i) edge-on orientation in which the polymer backbone lies perpendicular to the plane and the $\pi-\pi$ stacking parallel to the substrate; ii) face-one orientation in which the backbone lies parallel to the plane and the $\pi-\pi$ stacking perpendicular to the substrate ${ }^{1,27}$. The peaks at $0.37 \AA^{-1}, 0.75$ $\AA^{-1}$ and $1.14 \AA^{-1}$ correspond to the $<100>$ lamellar peak and its higher orders in the edge-on orientation, while the peak at $1.66 \AA^{-1}$ is consistent with the $<001>+<010>$ peak in a face-on lamellar orientation, and is indicative of a small fraction of lamellae adopting this geometry ${ }^{28}$. Note that this last peak could not be resolved in the neutron diffraction experiment carried out using the IRIS instrument ${ }^{1}$, because of the strong incoherent signal. If we now turn to the P3HT:PCBM blends ( $\left.2 b\right)$, we can see that the addition of PCBM leads to two main effects namely: i) the appearance of the PCBM crystalline peak at $1.36 \pm 0.1 \AA^{-1}$ and $1.47 \pm 0.1 \AA^{-1}$ that can be assigned to the $<221>$ and $<22-1>$ planes of the PCBM monoclinic structure, respectively ${ }^{7,29,30}$, and ii) the scattering intensity upturn seen at low-Q values $\left(<0.5 \AA^{-1}\right)$ that can be attributed to the scattering from the mixed P3HTPCBM amorphous domains ${ }^{31}$. The parabolic shape of the incoherent signal in the P3HT-PCBM blend 
does not follow the usual decaying profile as a function of momentum transfer. This peculiar effect is not fully understood yet, and will be further analysed in more details in future works.

In general, the diffraction patterns indicate that the incoherent scattering intensity, which originates essentially from the polymer side-chains, does not change appreciably upon blending, whereas the addition of PCBM clearly enhances the coherent signal over the all diffraction pattern.

(a)

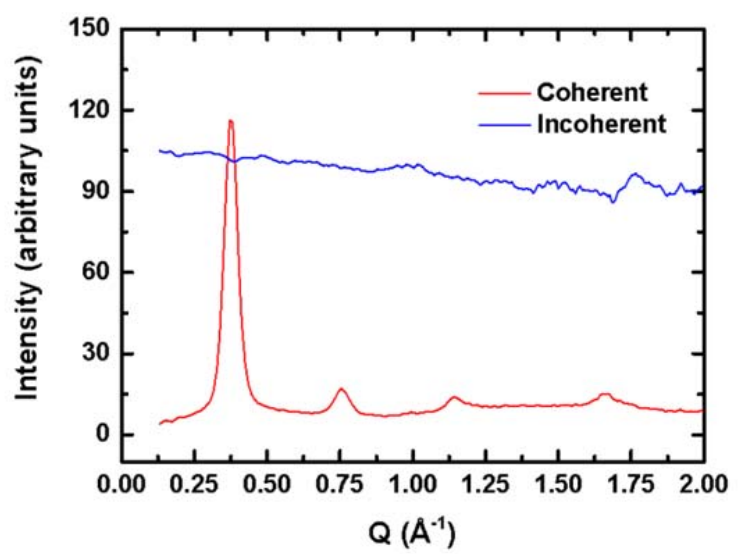

(b)

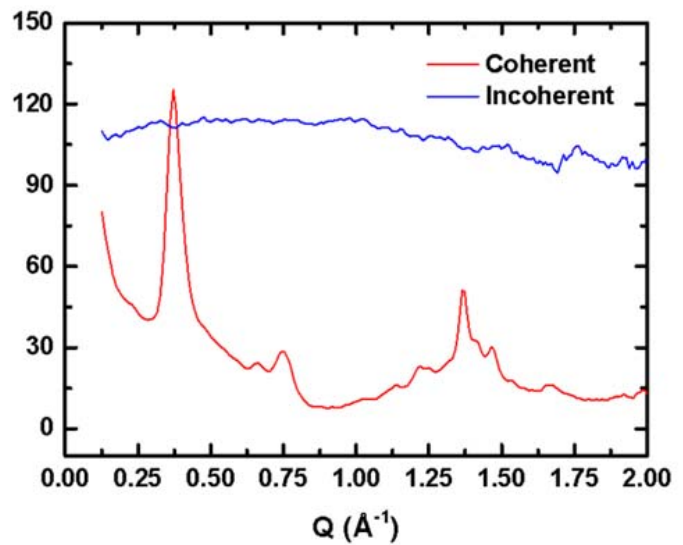

Figure 2: Coherent and incoherent contributions for P3HT (a) and P3HT-PCBM 1:1 blends cast from chloroform at 300K.

\subsection{Quasi-elastic incoherent neutron scattering}

We now turn to the study of the quasi-elastic signal. In figure 3, we shows a comparison between the incoherent and coherent signal intensity taken at $Q=1.2 \AA^{-1}$ and $433 \mathrm{~K}$. We can observe that the incoherent signal intensity is markedly predominant in both P3HT (3a) and P3HT-PCBM blend (3b), but that the coherent contribution increases upon addition of PCBM (3b). In addition, we do not observe any quasi-elastic coherent broadening for both samples at this timescale and within an instrumental resolution of $0.1 \mathrm{meV}$ (figures $3 \mathrm{c}, \mathrm{d}$ ), thus we will focus on the analysis of the QENS incoherent signal. 


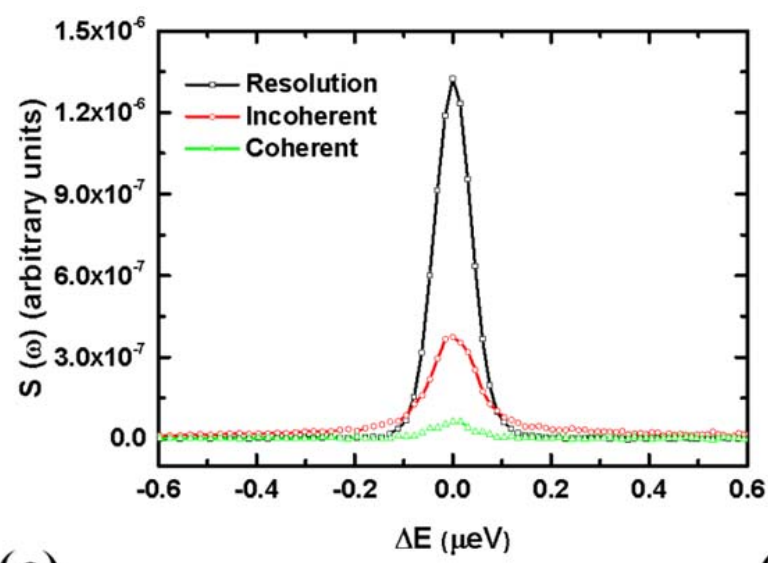

(c)

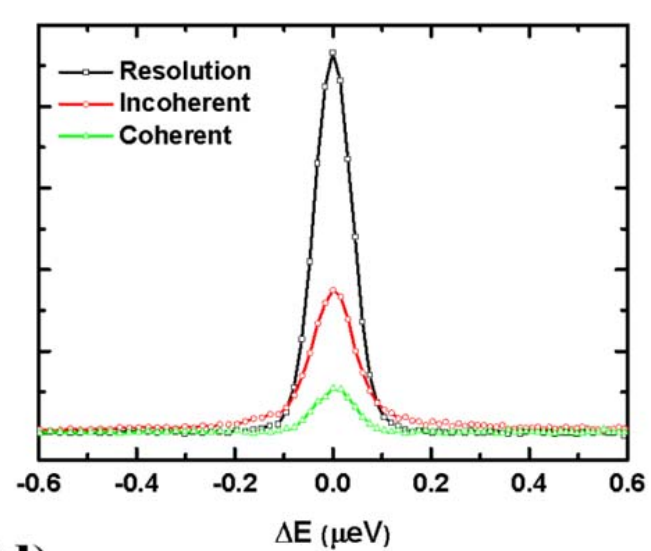

(d)
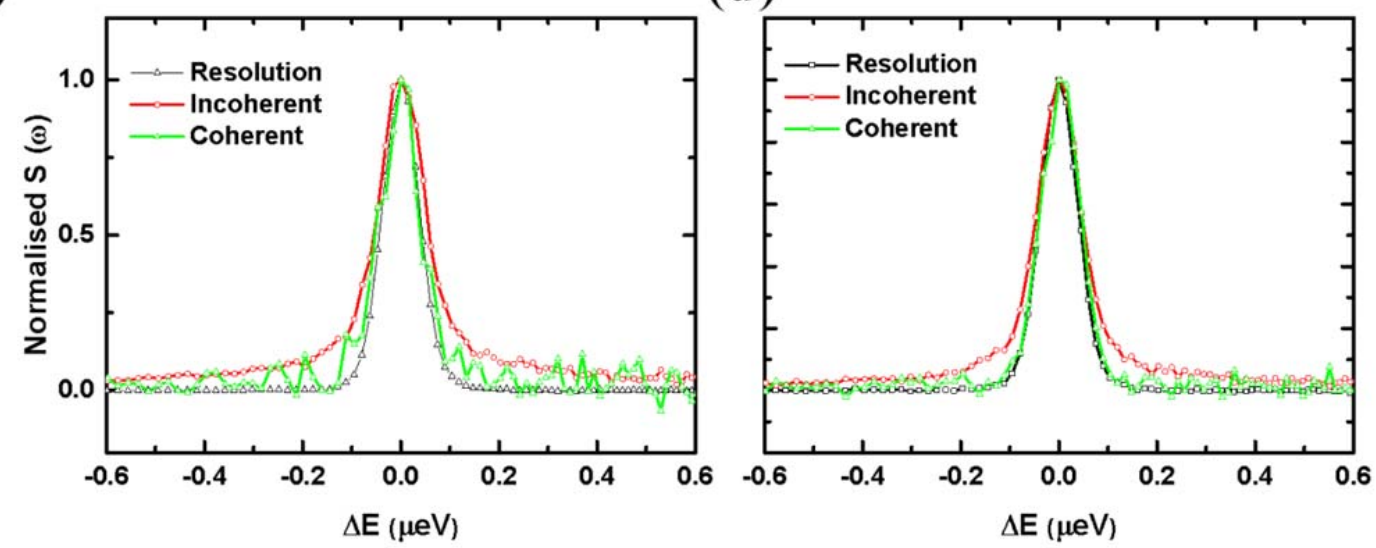

Figure 3: Incoherent and coherent dynamical structure factor for P3HT (a) and P3HT:PCBM (b), taken at $\mathrm{Q}=1.2 \AA^{-1}$ and $433 \mathrm{~K}$ for comparison. Normalised incoherent and coherent structure factor for P3HT (c) and P3HT:PCBM (d). The normalised plots indicate clearly that both the samples do not feature any coherent dynamics, as the $S(Q, \omega)_{\operatorname{coh}}$ almost overlaps the resolution line.

In figure 4a, we show the incoherent dynamical structure factor for pure P3HT, measured at three temperatures $(300 \mathrm{~K}, 363 \mathrm{~K}, 433 \mathrm{~K})$ in the $Q$-range $\left(0.3-1.8 \AA^{-1}\right)$. The shape of the spectra is a convolution of two main contributions: i) a delta function within the instrumental resolution due to elastic scattering and ii) a broadening due to the single particle motions. Depending on the dynamical properties of the sample and on the probed instrumental timescale, the broadening can be split into two or more Lorentzians, which can be indicative of different type of motions in different time and spatial domains. The incoherent dynamical structure factor spectra for 1:1 P3HT-PCBM blends seem to feature the same characteristics as those of the pure polymer (figure $4 b$ ), with the exception of a slightly less broad signal for the blend between $1.2-1.5 \AA^{-1}$ at $300 \mathrm{~K}$. This effect might be related to the slowing down of the polymer side-chain dynamics, likely due to polymer confinement within the lengthscale of PCBM crystalline domains. 
We fitted the spectra using a delta function, and two broad Lorentzians that account for faster $(\sim 1$ $\mathrm{meV})$ and slower $(\sim 0.1 \mathrm{meV})$ motions, all convoluted with the instrumental resolution measured at 2K. Figure 5 shows the elastic fraction and the FWHM of the two Lorentzians as a function of the momentum transfer $Q$. The elastic fraction plots suggest that the polymer side-chain dynamics are likely being constrained by confinement between the PCBM crystallites, as indicated by the slightly higher value of the elastic fraction at around the length scale of PCBM crystals. Unfortunately, the statistical uncertainty of the data, as can be seen from the quasi-flat and erratic trend of both the Lorentzians, does not enable us to gauge if their mobility is retarded and we cannot carry out a more complete dynamical analysis on these samples. Furthermore the instrumental resolution of D7 $(\sim 0.1$ $\mathrm{meV})$ is poorer than that of IRIS $(\sim 0.02 \mathrm{meV})$, where polarisation analysis could give us further information for the longer timescale motions. 
(a)

P3HT

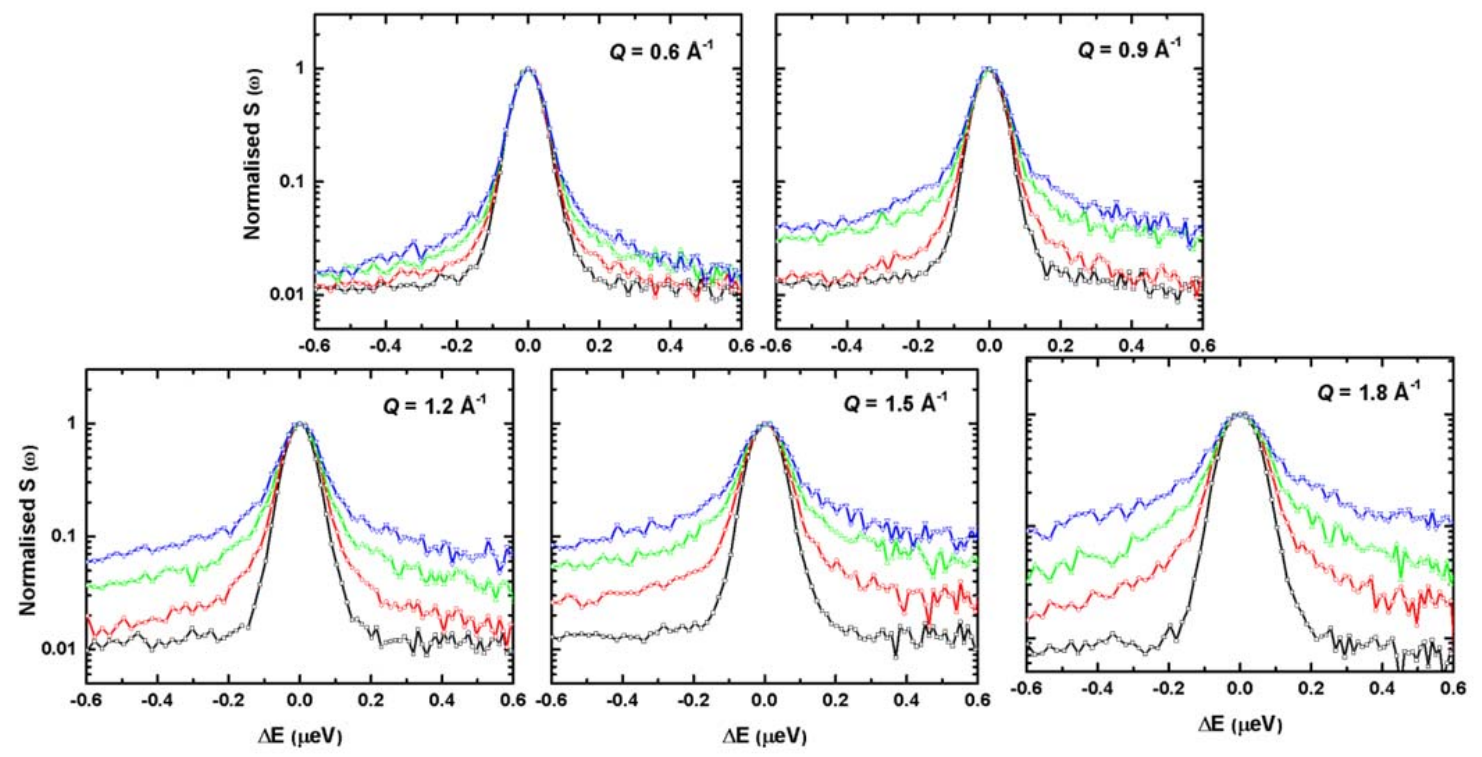

(b)

P3HT-PCBM
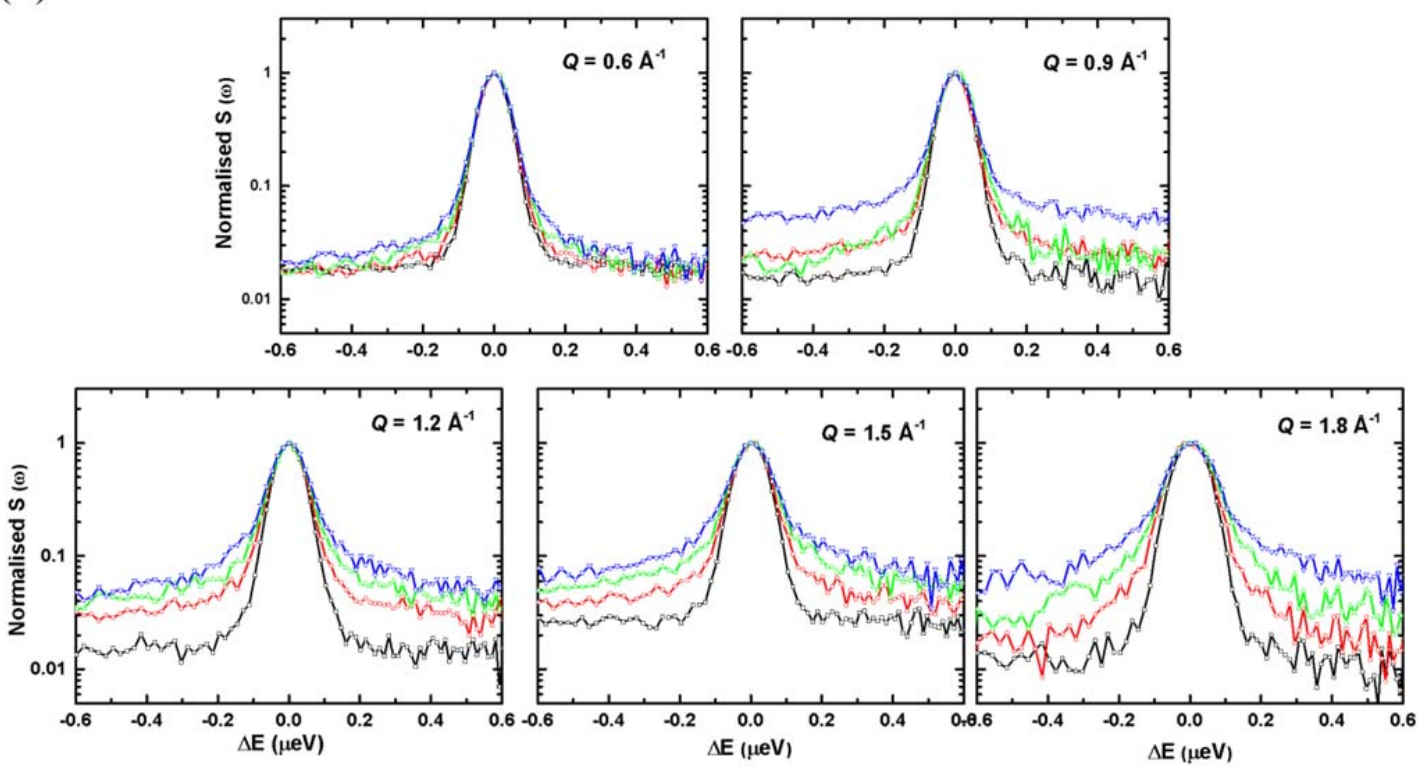

Figure 4: Incoherent dynamical structure factor determined from the spin-flip scattering for pure P3HT (a) and P3HTPCBM blends 1:1 blends (b), measured at three temperatures (black $2 \mathrm{~K}$, red $300 \mathrm{~K}$, green $363 \mathrm{~K}$, blue $433 \mathrm{~K}$ ) in the $0.6-1.8$ $\AA^{-1} Q$-range . All the spectra are reported in logarithmic scale and normalised to peak maximum (y-axis range $10^{-3}$ to 3 ) for comparison (y-axis range $5 \times 10^{-3}$ to 3 ). 

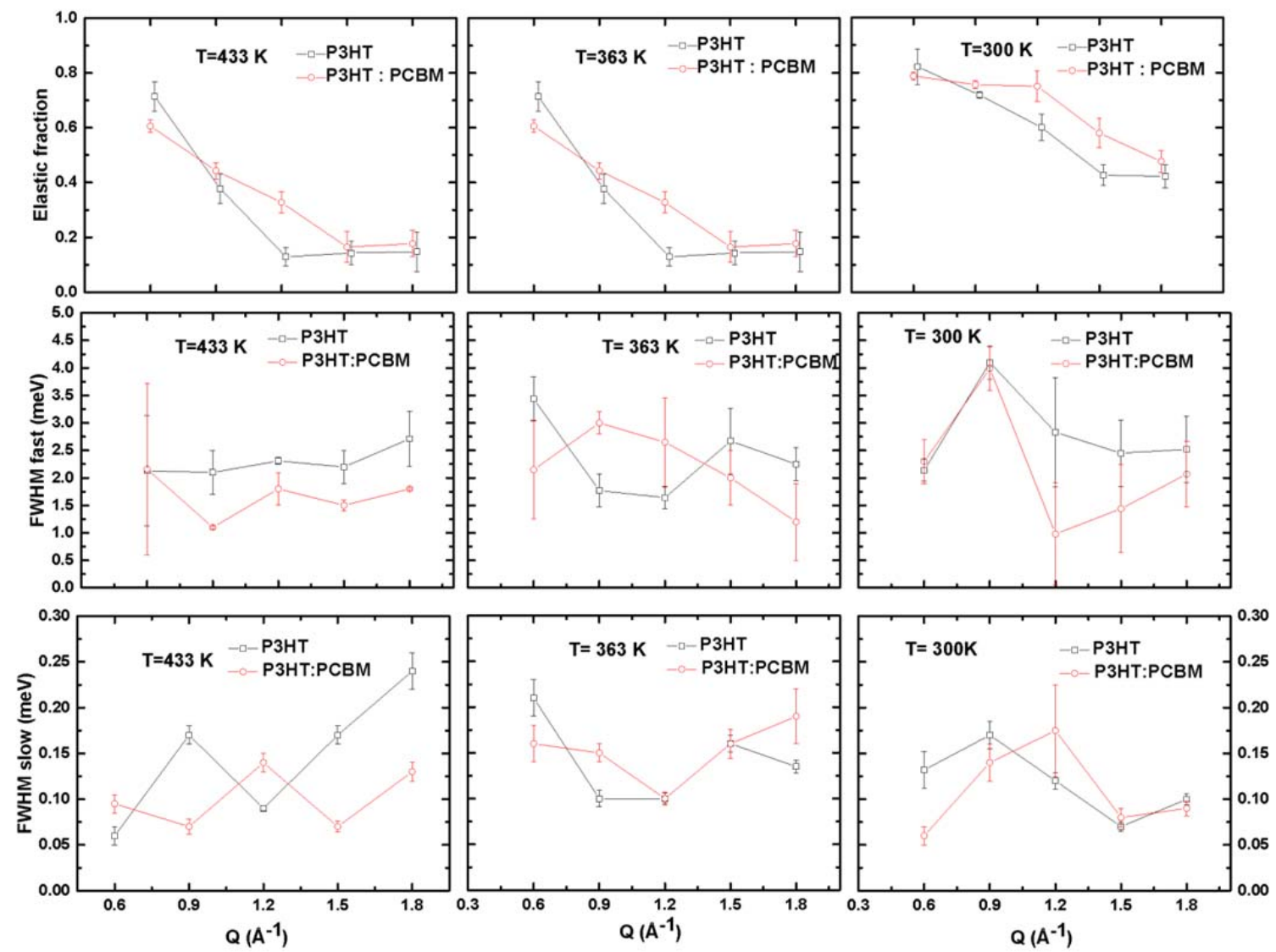

Figure 5: Elastic fraction of the pure polymer and P3HT-PCBM blend, as calculated from the ratio between the delta function area (elastic) and the two broads Lorentzians (inelastic), and the full width at half maximum (FWHM) of the Lorentzians as a function of the momentum transfer.

\section{Conclusions}

In this study, we performed an experimental separation of coherent and incoherent neutron scattering contributions on pure P3HT and P3HT: PCBM blends by means of neutron polarisation analysis. To the best of our knowledge this is the first time that this method has been applied on this class of functional materials. Neutron polarisation analysis is the method of choice to separate effectively the coherent collective scattering contribution from the incoherent single-particle scattering, especially when the time/length scales of the two events overlap. For instance, the excess of elastic signal at around the PCBM d-spacing $\left(1.4 \AA^{-1}\right)$ can make dynamical data interpretation at this length scale prone to error.

We showed that by removing the strong incoherent contribution of P3HT, we can disclose new structural coherent features, such as the partial face-on orientation of the polymer crystalline 
lamellae. Investigations of the pure incoherent QENS via the analysis of the dynamical structure factor, suggest that that motions of the polymer side-chain in the timescale of a few picoseconds are constrained and possibly being slowed down upon addition and further crystallisation of PCBM buckyballs.

Although we could not carry out a more in-depth dynamical analysis due to the low statistics of the data and the lower instrumental resolution of D7 compared with IRIS, we feel strongly that neutron polarisation analysis combined with QENS is a powerful technique to separate different kind of motions in complex systems which cannot be treated as purely incoherent or coherent and yield confidence in the data analysis and interpretation. In the case of polymer systems, this pertains to most systems, blends, nanocomposites, systems with very specialised polymers which are difficult to deuterate, biopolymers or those used for bio-applications - in all these cases the signal is a mixture of coherent and incoherent scattering and QENS with polarisation analysis would clear the ambiguity in the interpretation of both structural and dynamical neutron data. In the experiments presented in this work, we were able to use QENS with polarisation analysis at a resolution of $0.1 \mathrm{meV}$, but ideally we would like to be able to use the technique on neutron spectrometers of resolutions better suited to capture polymer glassy dynamics, in the hundreds of picoseconds and nanoseconds, such as backscattering spectrometers.

\section{Acknowledgements}

GMP is supported by an IMPACT PhD studentship co-sponsored by UCL and ISIS Pulsed Neutron and Muon Facility (Science and Technology Facilities Council) Student Agreement number 4070024391. We thank the EU Horizon 2020 Research and Innovation Programme under Grant Agreement No.643238 (SYNCHRONICS). The authors would also like to thank the Institut Laue Langevin, Grenoble, France, for access to the D7 instrument. FC is a Royal Society Wolfson Research Merit Award holder.

\section{References}

1. $\quad$ Paternó, G.; Cacialli, F.; García-Sakai, V. Chemical Physics 2013, 427, (0), 142-146.

2. $\quad$ Schweitzer, B.; Bassler, H. Synthetic metals 2000, 109, (1-3), 1-6.

3. Barford, W., Electronic and optical properties of conjugated polymers. Oxford University Press: 2005.

4. Halls, J. J. M.; Walsh, C. A.; Greenham, N. C.; Marseglia, E. A.; Friend, R. H.; Moratti, S. C.; Holmes, A. B. Nature 1995, 376, (6540), 498-500. 
5. Yu, G.; Gao, J.; Hummelen, J. C.; Wudl, F.; Heeger, A. J. Science 1995, 270, (5243), 17891791.

6. $\quad$ Wang, H.; Liu, J.; Han, Y. Polymer 2013, 54, (2), 948-957.

7. Paternò, G.; Warren, A. J.; Spencer, J.; Evans, G.; García Sakai, V.; Blumberger, J.; Cacialli, F. Journal of Materials Chemistry C 2013, 1, (36), 5619-5623.

8. Lazzerini, G. M.; Paternò, G. M.; Tregnago, G.; Treat, N.; Stingelin, N.; Yacoot, A.; Cacialli, F. Applied physics letters 2016, 108, (5), 053303.

9. Tregnago, G.; Wykes, M.; Paternò, G. M.; Beljonne, D.; Cacialli, F. The Journal of Physical Chemistry C 2015, 119, (21), 11846-11851.

10. Yin, W.; Dadmun, M. ACS nano 2011, 5, (6), 4756-68.

11. Chen, D.; Liu, F.; Wang, C.; Nakahara, A.; Russell, T. P. Nano letters 2011, 11, (5), 2071-8.

12. Kiel, J. W.; Kirby, B. J.; Majkrzak, C. F.; Maranville, B. B.; Mackay, M. E. Soft Matter 2010, 6, (3), 641-646.

13. Li, G.; Zhu, R.; Yang, Y. Nature Photonics 2012, 6, (3), 153-161.

14. García Sakai, V.; Arbe, A. Curr Opin Colloid In 2009, 14, (6), 381-390.

15. Obrzut, J.; Page, K. A. Phys Rev B 2009, 80, (19).

16. Guilbert, A. A. Y.; Urbina, A.; Abad, J.; Diaz-Paniagua, C.; Batallan, F.; Seydel, T.; Zbiri, M.; Garcia-Sakai, V.; Nelson, J. Chemistry of Materials 2015, 27, (22), 7652-7661.

17. Díaz-Paniagua, C.; Urbina, A.; García-Sakai, V.; Seydel, T.; Abad, J.; Padilla, J.; GarcíaValverde, R.; Espinosa, N.; Gómez-Escalonilla, M. a.-J.; Langa, F.; Batallán, F. Chemical Physics 2013, 427, 129-141.

18. Etampawala, T.; Ratnaweera, D.; Morgan, B.; Diallo, S.; Mamontov, E.; Dadmun, M. Polymer 2015, 61, 155-162.

19. Carlile, C. J.; Adams, M. A. Physica B 1992, 182, (4), 431-440.

20. Burankova, T.; Hempelmann, R.; Wildes, A.; Embs, J. P. The journal of physical chemistry. $B$ 2014, 118, (49), 14452-60.

21. Stewart, J. R.; Deen, P. P.; Andersen, K. H.; Schober, H.; Barthélémy, J. F.; Hillier, J. M.; Murani, A. P.; Hayes, T.; Lindenau, B. Journal of Applied Crystallography 2008, 42, (1), 69-84.

22. $\quad$ Kropka, J. M.; Garcia Sakai, V.; Green, P. F. Nano Lett 2008, 8, (4), 1061-5.

23. Stewart, J. R.; Deen, P. P.; Andersen, K. H.; Schober, H.; Barthelemy, J. F.; Hillier, J. M.; Murani, A. P.; Hayes, T.; Lindenau, B. J Appl Crystallogr 2009, 42, 69-84.

24. Moon, R. M.; Riste, T.; Koehler, W. C. Physical Review 1969, 181, (2), 920-931.

25. Richard, D. F., M.; Kearley, G. J.; Bradley, A. D. The LAMP book 2013, Institut LaueLangevin (Grenoble, France), http://www.ill.eu/?id=2024.

26. Azuah, R. T.; Kneller, L. R.; Qiu, Y. M.; Tregenna-Piggott, P. L. W.; Brown, C. M.; Copley, J. R. D.; Dimeo, R. M. Journal of Research of the National Institute of Standards and Technology 2009, 114, (6), 341-358.

27. Sirringhaus, H.; Brown, P. J.; Friend, R. H.; Nielsen, M. M.; Bechgaard, K.; Langeveld-Voss, B. M. W.; Spiering, A. J. H.; Janssen, R. A. J.; Meijer, E. W.; Herwig, P.; de Leeuw, D. M. Nature 1999, 401, (6754), 685-688.

28. Li Destri, G.; Keller, T. F.; Catellani, M.; Punzo, F.; Jandt, K. D.; Marletta, G. Macromolecular Chemistry and Physics 2011, 212, (9), 905-914.

29. Rispens, M. T.; Meetsma, A.; Rittberger, R.; Brabec, C. J.; Sariciftci, N. S.; Hummelen, J. C. Chem Commun 2003, (17), 2116-2118.

30. Destri, G. L.; Keller, T. F.; Catellani, M.; Punzo, F.; Jandt, K. D.; Marletta, G. Langmuir 2012, 28, (11), 5257-66.

31. Chen, C. Y.; Tsao, C. S.; Huang, Y. C.; Liu, H. W.; Chiu, W. Y.; Chuang, C. M.; Jeng, U. S.; $\mathrm{Su}, \mathrm{C}$. J.; Wu, W. R.; Su, W. F.; Wang, L. Nanoscale 2013, 5, (16), 7629-38. 